\title{
Sedimentação da Lagoa Itapeva, RS, Brasil
}

\author{
Michel Doeppre IVANOFF ${ }^{1}$, Elírio Ernestino TOLDO Jr. ${ }^{1}$ \& Rubens Cesar Lopes FIGUEIRA²
}

1. Centro de Estudos de Geologia Costeira e Oceânica, Instituto de Geociências, Universidade Federal do Rio Grande do Sul. Av. Bento Gonçalves, 9500, CEP 91501-970, Porto Alegre, RS, Brasil. E-mail: michel.ivanoff@ufrgs.br, toldo@ufrgs.br.

2. Departamento de Oceanografia Física, Química e Geológica, Universidade de São Paulo. Praça do Oceanográfico, 191, Cidade Universitária, CEP 05508-120, São Paulo, SP, Brasil. E-mail: rfigueira@usp.br.

Recebido em 02/2013. Aceito para publicação em 11/2013.

Versão online publicada em 04/04/2014 (www.pesquisasemgeociencias.ufrgs.br)

\begin{abstract}
Resumo - Os fatores hidrodinâmicos que controlam a sedimentação na Lagoa Itapeva foram avaliados através do mapa de distribuição granulométrica gerado a partir do diagrama de Pejrup e da análise da ação hidrodinâmica induzida pelas ondas no corpo lacustre. Os padrões texturais das amostras de fundo indicaram a presença de significativa energia hidrodinâmica atuante no processo de sedimentação, com predomínio da fração arenosa nas margens submersas e granodecrescência para tamanhos silte grosso a fino em direção ao centro. 0 teor de matéria orgânica também apresenta este padrão, com aumento gradativo na área central do corpo lacustre e nas proximidades das desembocaduras dos rios Três Forquilhas e Cardoso. Os principais processos que condicionam a sedimentação na lagoa são controlados pelo aporte de sedimentos fluviais, redistribuídos pela ação das ondas e correntes induzidas pela ação dos ventos. A concentração de grãos tamanho argila é pouco significativa, devido à baixa inserção pelos rios, além da agitação gerada por ondas sobre o piso lacustre, que dificulta a deposição desta classe granulométrica. A presença de sedimentos finos nos locais mais profundos e nas áreas mais abrigadas indica a influência de correntes menos efetivas, permitindo a deposição nestes locais. Palavras-chave: sedimentação lacustre, hidrodinâmica lacustre, Lagoa Itapeva.
\end{abstract}

Abstract - Sedimentation of ItAPEVA LAKE, RS, BRAziL. Hydrodynamic factors that control sedimentation in the Itapeva Lake were assessed on the granulometric distribution map generated by the Pejrup's diagram and by the analysis of the hydrodynamic action, which results from the waves in the lacustrine body. The texture patterns of bottom samples showed that there is significant hydrodynamic energy in the sedimentation process: the sand fraction prevails in the submerged margins whereas grain size decreases, from coarse silt to fine silt, moved towards the center. The content of organic matter also shows this pattern, with a steady increase in the central area of the lacustrine body and near the mouths of the Três Forquilhas and Cardoso rivers. The main processes that determine sedimentation are mainly controlled by the amount of fluvial sediment, which is redistributed by the action of waves and currents resulting from the action of winds. The concentration of clay-sized grains is not very significant not only because there is low input by the rivers, but also because the wave agitation avoid this granulometric class from depositing on the lacustrine bottom. Fine sediment in the deepest spots and in the most sheltered areas shows the influence of less effective currents that enable deposition in these areas.

Key words: lacustrine sedimentation, lacustrine hydrodynamics, Itapeva Lake.

\section{Introdução}

Lagoas costeiras são corpos aquosos relativamente rasos, separados do oceano por barreiras arenosas, ou em alguns casos conectadas a ele por um ou mais canais restritos. Muitas lagoas e seus depósitos associados são resultantes da variação do nível do mar durante o Quaternário e da construção de barreiras por processos marinhos, que isolam parcial ou totalmente os corpos lagunares (Kjerfve et al., 1997).

A evolução destes corpos costeiros em grande parte é determinada por ciclos, de forma que o seu desenvolvimento deriva de uma baía rasa aberta ou de um vale estuarino parcialmente isolado. Devido ao preenchimento progressivo por sedimentos, tais cor- pos evoluem a pântanos ou depósitos deltaicos. Desta forma, seu término normalmente é determinado por deposição sedimentar ou pelo aumento do nível relativo do mar, ocasionando destruição por erosão marinha (Nichols, 1989). Devido a estabilidade temporária do nível do mar, estes ambientes representam produtos momentâneos do ponto de vista geológico e de processos de sedimentação costeira.

Durante o século passado, muitas regiões litorâneas e áreas no entorno de lagoas costeiras atraíram grandes núcleos populacionais e, desta forma, tornaram-se ameaçadas pelos resíduos emitidos pelas indústrias e pelos núcleos urbanos. Além disso, as atividades antrópicas alteram drasticamente o fornecimento de sedimentos nestes ambientes, através da construção de 
barragens ou utilização de bacias hidrográficas para inserção da agricultura.

A análise das características da dinâmica sedimentar é essencial ao gerenciamento destes sistemas costeiros. Processos dinâmicos como erosão, transporte e deposição criam feições morfológicas e estruturas peculiares, de forma que o entendimento desses mecanismos, observados os distintos processos presentes, fornece subsídios para avaliar a evolução destes ambientes sedimentares.

Sendo assim, o presente trabalho tem por objetivo caracterizar a composição textural e distribuição dos sedimentos do piso lacustre, bem como a ação hidrodinâmica provocada pela ação das ondas associada à morfologia de fundo, contribuindo, desta forma, para um melhor entendimento da dinâmica sedimentar destes ambientes costeiros modernos.

\section{Localização e área de estudo}

A Lagoa Itapeva $\left(29^{\circ} 30^{\prime} \mathrm{S} / 49^{\circ} 40^{\prime} \mathrm{W}\right)$ é a primeira de uma série de lagoas costeiras, no sentido norte - sul, paralelas à linha de costa no litoral norte do Rio Grande do Sul, delimitada pelas escarpas da Serra Geral a Oeste e pelo Oceano Atlântico a Leste. A lagoa tem formato alongado, apresentando orientação NE-SW com 32 km de comprimento e largura média que varia de $2,5 \mathrm{~km}$ na porção NE a 5,5 km na porção SW e uma área de aproximadamente $125 \mathrm{~km}^{2}$ (Fig. 1).

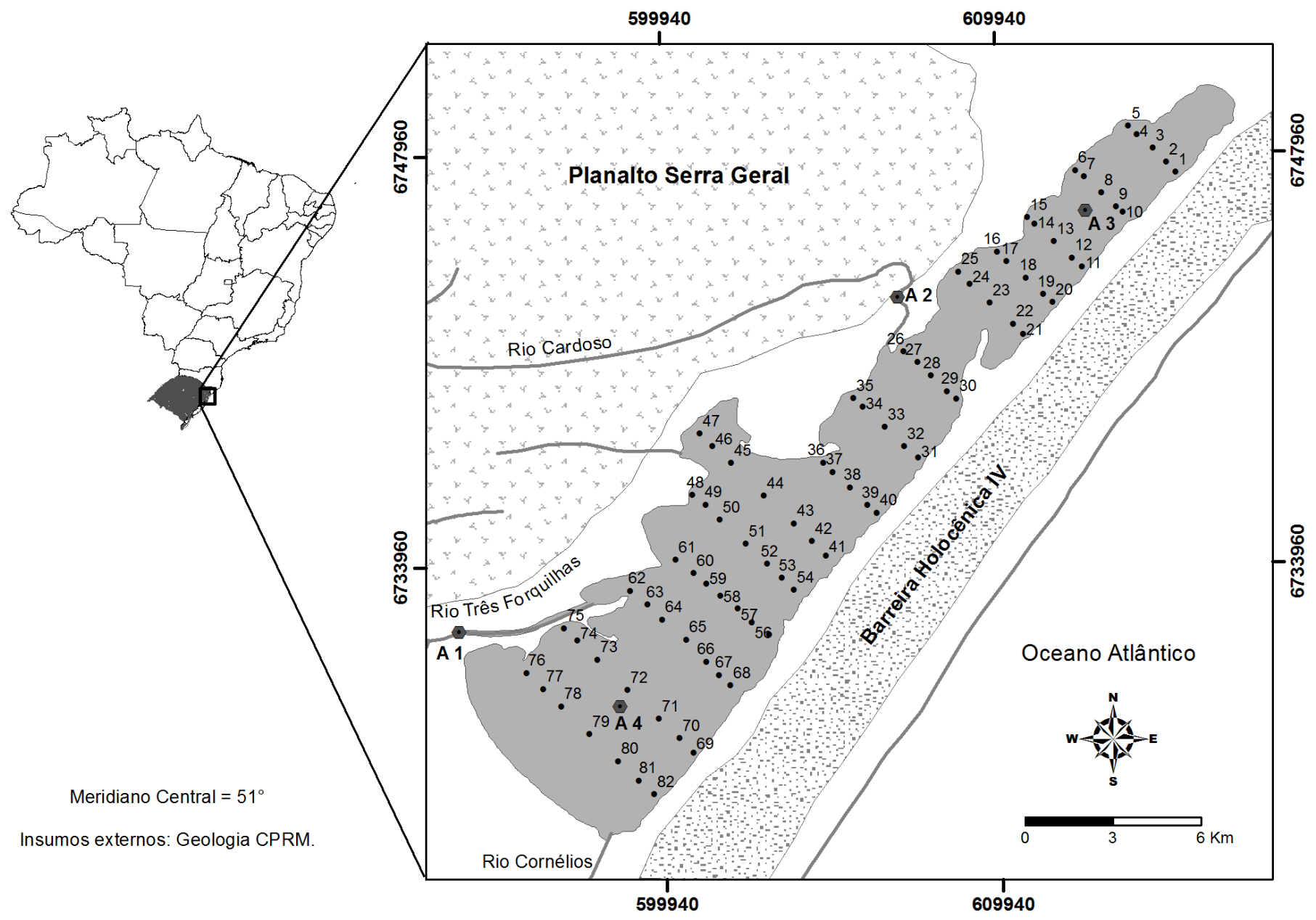

Figura 1. Mapa da área de estudo com ênfase na Lagoa Itapeva inserida na planície costeira do Rio Grande do Sul-Brasil, contendo a localização dos pontos de amostragem de sedimentos de fundo.

O desenvolvimento dos sistemas lacustres presentes na Planície Costeira do Estado do Rio Grande do Sul ocorreu durante os eventos transgressivo-regressivos provocados pelas oscilações eustáticas do nível do mar nos últimos $400 \mathrm{Ka}$. Como consequência dessas variações, foram formados quatro sistemas deposicionais Laguna-Barreira, designados do mais antigo para o mais recente, como sistemas Laguna-Barreira I, II, III de idade pleistocênica e a Barreira IV, de idade holocênica (Villwock, 1984).
A Lagoa Itapeva está inserida no espaço de retrobarreira do sistema deposicional Laguna Barreira IV, tendo sua gênese vinculada ao final da Última Grande Transgressão, que inundou a área pertencente ao compartimento da lagoa há aproximadamente 6,5 Ka AP (Villwock \& Tomazelli, 1998).

A história do comportamento do nível do mar relacionado à Transgressão Marinha Pós-glacial (TMP) no Rio Grande do Sul se inicia em 17,5 Ka AP quando este nível situava-se a cerca de 120-130 m abaixo do atual 
(Corrêa \& Toldo Jr., 1998). Desde então, o nível do mar subiu rapidamente até cerca de 6,5 Ka AP, a uma taxa média de 1,2 cm.ano ${ }^{-1}$. Entre 5 - $6 \mathrm{Ka}$, alcançou sua altitude máxima na costa sul e leste brasileira, mostrando, desde então, uma tendência geral de rebaixamento até o presente (Martin et al., 1979; Angulo \& Lessa, 1997).

No Rio Grande do Sul, estima-se que o nível do mar atingiu altitudes máximas entre 2 e $4 \mathrm{~m}$, formando um grande sistema lagunar. A partir de então, se estabeleceu na costa do estado uma fase predominantemente regressiva, condicionada pelo rebaixamento gradual do NRM até a cota atual, associado a um balanço positivo de sedimentos (Dillenburg et al., 2000, 2006). 0 rebaixamento do NRM proporcionou condições para um acelerado processo de assoreamento e segmentação do grande sistema lagunar existente, e este processo isolou parte do sistema lagunar que atualmente é representado pela Lagoa Itapeva.

O clima da área de estudo, segundo a classificação de Koppen-Geiger apresentada por Strahler \& Strahler (1987), é a do tipo $C f a$, o qual caracteriza um clima temperado chuvoso, com precipitações distribuídas regularmente ao longo do ano e temperatura média do mês mais quente superior a $22^{\circ} \mathrm{C}$ e a do mês mais frio superior a $-3^{\circ} \mathrm{C}$. Segundo Hasenack \& Ferraro (1989), o clima do litoral norte apresenta uma temperatura média anual de $20^{\circ} \mathrm{C}$, taxa de precipitação e evaporação anual de 1322 e 1134 mm. a $^{-1}$ respectivamente, e uma umidade relativa média de $83 \%$.

A costa do Rio Grande do Sul está submetida a um regime de micro-marés com amplitude média de aproximadamente $0,25 \mathrm{~m}$, controladas por fatores astronômicos; durante o inverno, no entanto, há significativa ocorrência de marés meteorológicas, associadas a tempestades, resultando em sobre-elevações do nível do mar de até 1,23 m (Toldo Jr. et al., 2000). Os ventos predominantes vêm do quadrante NE (Schwarzbold \& Schafer, 1984; Medeiros, 1992) e são constantes ao longo do ano, porém mais intensos na primavera-verão; no outono-inverno, são mais constantes os ventos de S e SW.

\section{Materiais e métodos}

Para a realização deste trabalho, foram obtidas 82 amostras de sedimentos superficiais de fundo (Fig. 1) coletadas em fevereiro de 2011 com um amostrador do tipo Van Veen. As amostras foram analisadas segundo o método de peneiramento à úmido em malha de 0,062 mm para a separação dos sedimentos lamosos $(<0,062$ $\mathrm{mm})$ das classes arenosas $(>0,062 \mathrm{~mm})$. A fração arenosa retida na malha voltou à estufa para secagem e posterior análise mecânica por peneiramento com intervalos de $1 \Phi$. Foi utilizado o método de pipetagem, para as classes de intervalos inferiores a este diâmetro, na determinação da fração de silte e argila (Krumbein \& Pettijohn, 1938; McManus, 1988).

As frações retidas em cada peneira, juntamente com os dados da pipetagem, foram pesadas, e os resultados analisados no software de processamento estatístico e textural PANCON, descrito por Toldo Jr. \& Medeiros (1986), para obtenção dos parâmetros estatísticos de Folk \& Ward (1957).

A partir do cálculo das frequências simples e acumuladas, estes valores foram importados para o Software SYSGRAN ${ }^{\circledR}$ (Camargo, 2006), para a representação dos resultados das amostras no diagrama triangular de Pejrup (Fig. 2).

0 diagrama de Pejrup constitui uma maneira de avaliar as condições dos locais onde as amostras foram tomadas, utilizando a concentração de argila para interpretar a energia hidrodinâmica presente no sistema atuante no processo de transporte e deposição dos sedimentos (Pejrup, 1988).

A confecção das curvas batimétricas e das curvas de isoteores foi efetuada através do programa ArcGIS 10, utilizando o método de "Krigagem" na interpolação dos pontos.
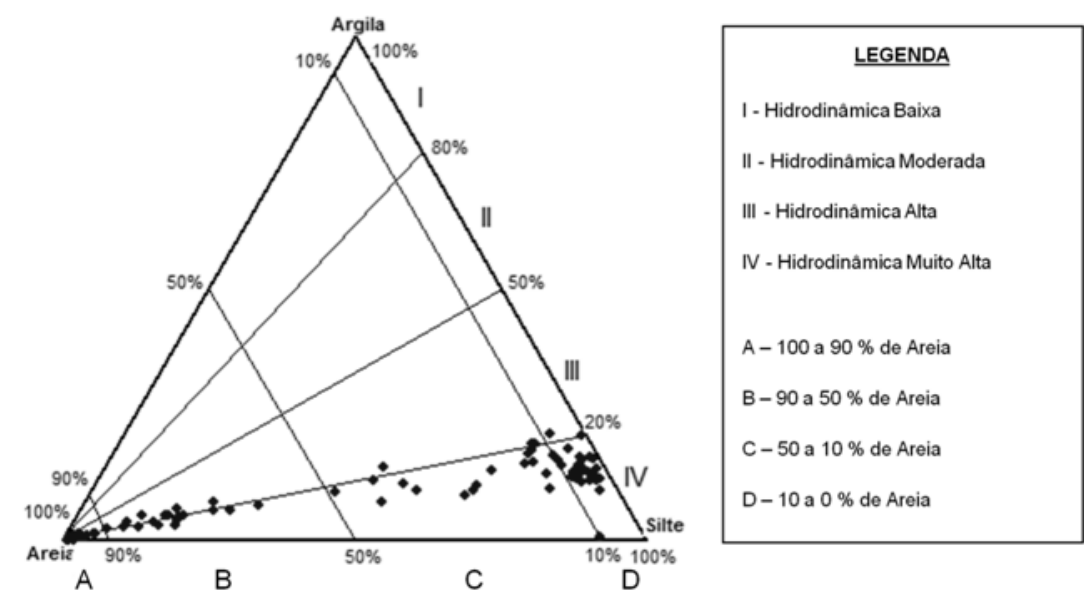

Figura 2. Diagrama triangular com a representação das amostras de fundo da Lagoa Itapeva, modificado de Pejrup (1988). 
A determinação do teor de matéria orgânica total foi obtida por meio de queima de $3 \mathrm{~g}$ de sedimento em mufla, a $550^{\circ} \mathrm{C}$ por $4,5 \mathrm{~h}$. A relação percentil entre o peso inicial (anterior à queima) e o peso final (após a queima) corresponde ao teor de matéria orgânica total da amostra, conforme descrito por Wetzel (1975), sendo determinada pela expressão:

$$
\% \text { M. O. }=\frac{P \cdot A \cdot \times 100}{P \cdot a .}
$$

onde, \% M.O. é o percentual de matéria orgânica, P.A. é o peso da amostra queimada e P.a. o peso da amostra antes da queima.

A determinação da granulometria dos sedimentos fluviais que ingressam na Lagoa Itapeva, foi realizada com granulômetro da série Cilas, modelo 1180 , em agosto de 2012. Foram coletadas amostras de água com garrafa Van Dorn nos rios Três Forquilhas (A1) e Cardoso (A2), bem como no interior da Lagoa Itapeva, nos setores norte (A3) e sul (A4), sendo as amostras obtidas em superfície, meio e fundo (Fig. 1).

\section{Resultados e discussões}

\subsection{Padrões texturais e morfologia de fundo}

A análise granulométrica é considerada um parâmetro essencial para se compreender o ambiente de sedimentação e o regime hidrodinâmico a que um ecossistema encontra-se submetido. 0 tamanho dos grãos, desde as frações mais grossas (cascalho) às frações mais finas (silte e argila), oferece informações quanto ao diâmetro médio e grau de seleção dos sedimentos, ou seja a energia de transporte a qual o ambiente é submetido, bem como a variabilidade desta.

A superfície, a forma e a profundidade da bacia afetam a circulação interna, influenciando as condições hidrodinâmicas em lagoas, sendo de grande importância na análise da distribuição e acumulação de sedimentos. Tal concepção serve de guia para identificar os processos sedimentares atuantes em seu interior, bem como avaliar as condições físicas, químicas e biológicas em lagoas (Schäfer, 1988).

Os resultados referentes à interpolação das cotas batimétricas demonstraram que a morfologia de fundo da Lagoa Itapeva apresenta profundidade reduzida, não ultrapassando $2,70 \mathrm{~m}$, com suave inclinação do piso lacustre em direção leste (Fig. 3).

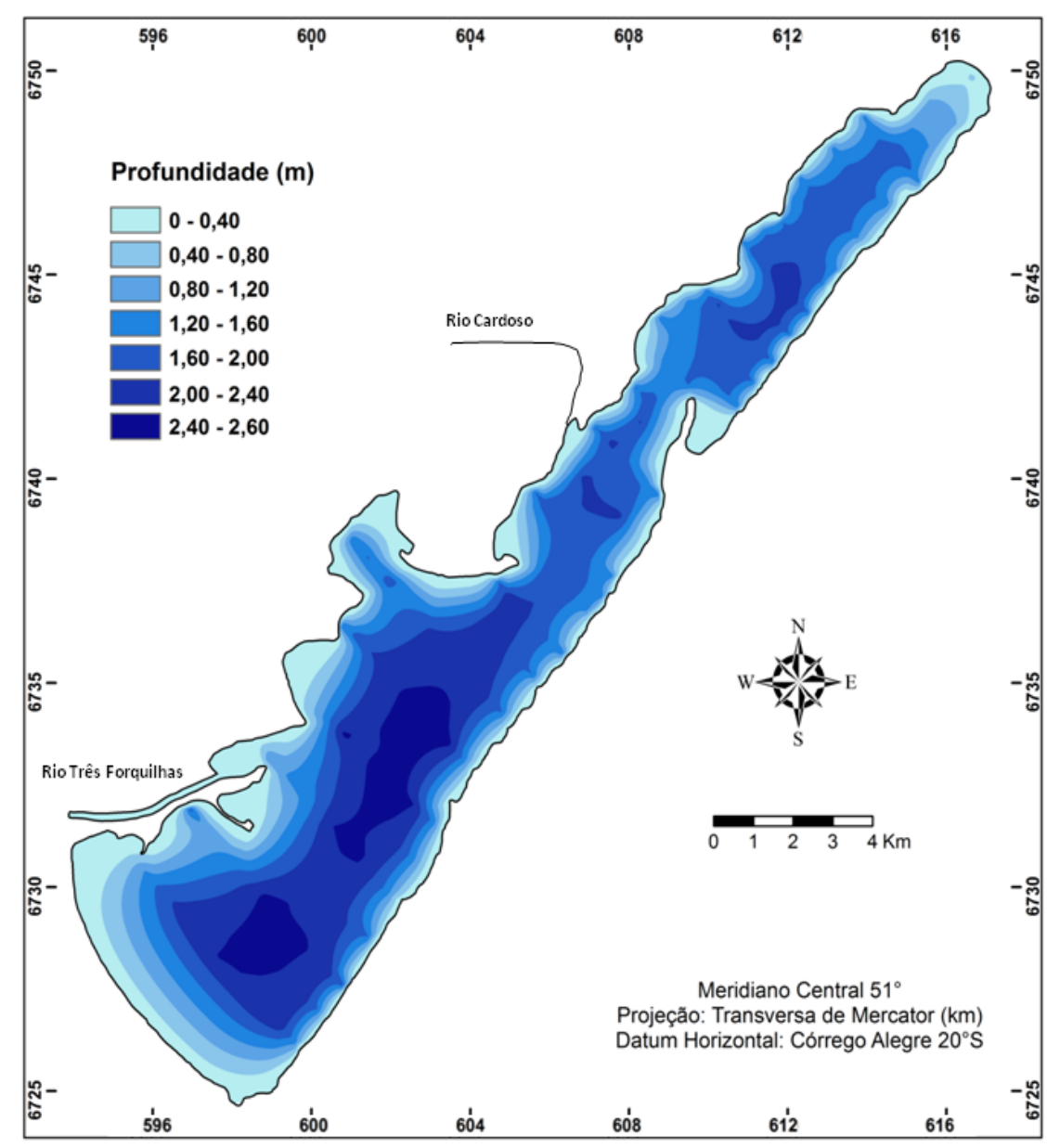

Figura 3. Mapa batimétrico da Lagoa Itapeva. 
A inclinação registrada a leste é comum a outras lagoas costeiras do estado, segundo trabalhos realizados por Tabajara (1994), Toldo Jr. et al. (2000) e Meyer et al. (2006). Esta característica é condicionada pela declividade da superfície pleistocênica de sub-fundo segundo estudos de geofísica realizados na Lagoa dos Patos por Toldo Jr. et al. (2006) - e pela influência de sedimentação eólica relativa à presença de dunas transgressivas nas margens leste lacustres. Devido a este processo de colmatação, muitos destes corpos costeiros já sofreram grandes modificações nas suas morfologias iniciais, com expressiva redução de seus tamanhos originais (Tomazelli \& Villwock, 1991).

Com base nos resultados granulométricos das amostras de fundo (Fig. 4), foi possível identificar quatro padrões de fácies, levando em consideração o teor de areia presente nas amostras proposto por Folk (1968): fácies arenosa (teor de areia acima de 90\%), fácies areno-lamosa (teor de areia entre 50 e 90\%), fácies lamo-arenosa (teor de areia entre 10 e 50\%) e fácies lamosa (teor de areia inferior a 10\%).

A fácies arenosa corresponde a um fundo erosivo, inserida no grupo A-IV no diagrama de Pejrup (Fig. 2), sendo composta por sedimentos não coesivos, com concentração de areia acima de $90 \%$ e teor de matéria orgânica inferior a 4\% (Fig. 5). Correspondem às áreas rasas da lagoa com ação hidrodinâmica muito alta, onde as ondas promovem um maior nível energético, impedindo a deposição de sedimentos finos. Estes sedimentos são compostos por areias quartzosas finas a muito finas, com grau de seleção que varia de moderada a bem selecionado e com tendência a assimetria positiva.

A ocorrência de areia muito grossa a grânulos fica restrita à porção deltaica do rio Três Forquilhas. As margens apresentam grande quantidade de sedimento biogênico, com exemplares de Heleobia sp. de granulometria na classe areia muito grossa e das espécies Anodontites trapesialis e Corbicula fluminea, de tamanho cascalho.

Deste modo, é possível verificar a ocorrência de sedimentos arenosos nas margens da lagoa até a isóbata de 1,6 m. No entanto, o setor próximo ao pontal na margem leste exibe pouca profundidade no sentido sudeste - noroeste, determinando a ocorrência de sedimentos arenosos que cruzam o fundo desta área (Fig. 4).

Neste local, observa-se uma feição embrionária de segmentação típica de corpos lagunares e lacustres, sujeitos a um padrão de ventos bidirecionais (Zenkovitch, 1959). A contínua ação das ondas geradas pelos ventos nestes corpos d'água desenvolve o crescimento de esporões pela variação espacial do fluxo de sedimentos quando paralelos à linha de costa.

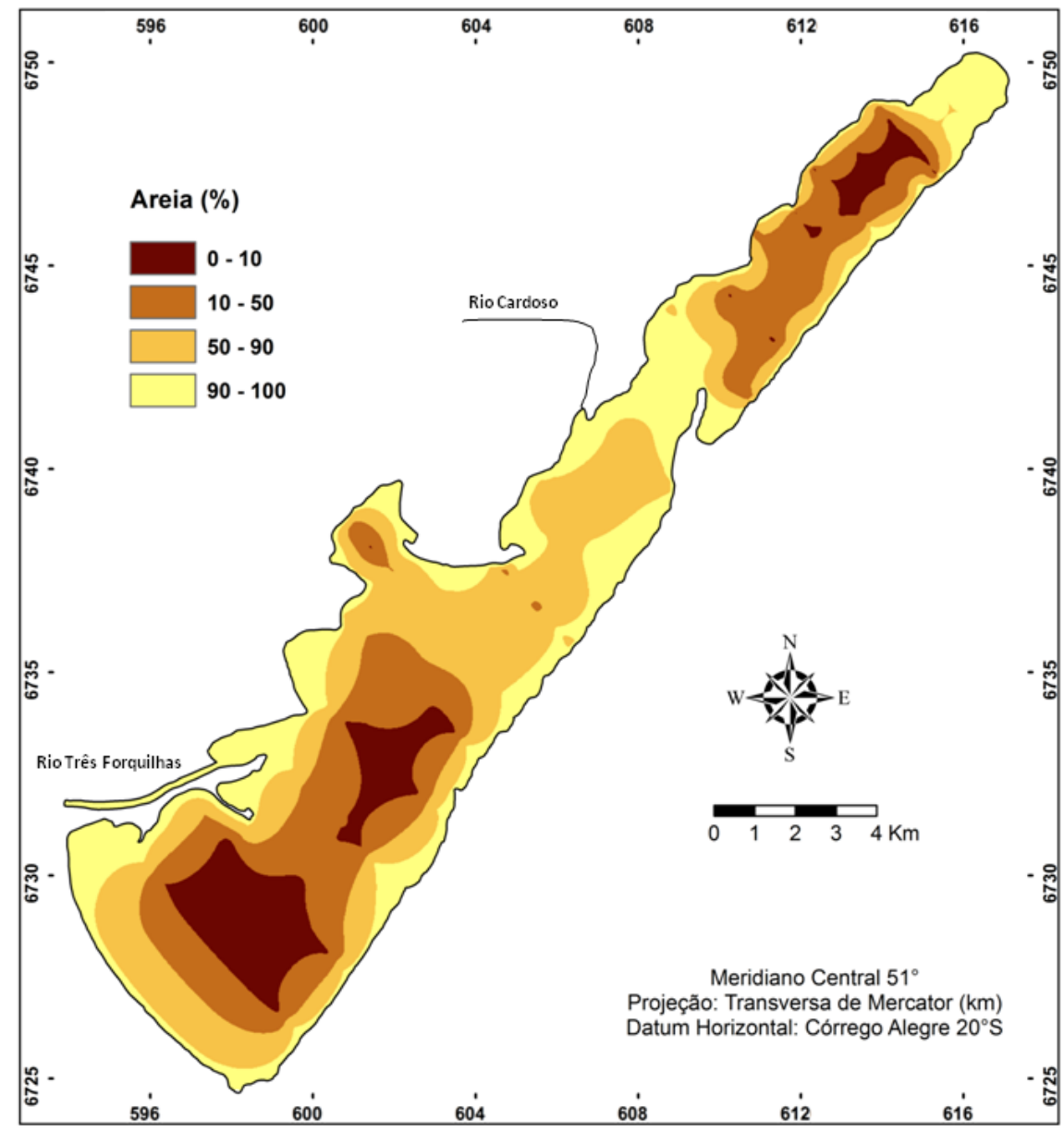

Figura 4. Mapa textural com os percentuais de areia da Lagoa Itapeva. 
Os esporões são saliências na linha de costa em forma de cúspides, formados por material não coesivo. Estas feições geomorfológicas são comuns em lagunas de todo o mundo, e no Brasil os principais exemplos são encontrados na Lagoa dos Patos (RS), como descrito por Toldo Jr. (1998), e na Laguna de Araruama (RJ), descrito por Alves et al. (2006).

A fácies areno-lamosa corresponde a um fundo transicional, com intervalo de concentração de areia entre 50 a $90 \%$ (Fig. 4). Nesta área, além da isóbata de 1,6 m, predominam grãos de diâmetros entre areia muito fina a silte médio, caracterizando um local de energia hidrodinâmica alta, classificada como grupo B (BIII e BIV), proposto por Pejrup (1988). Estes sedimentos apresentam seleção que varia de moderada a pobremente selecionada e assimetria que oscila entre aproximadamente simétrica a positiva. É possível constatar, neste fundo, um teor de matéria orgânica em torno de 6 a $8 \%$.

As fácies lamo-arenosa e lamosa correspondem a um fundo deposicional com intervalos de concentração de areia entre 10 a 50\%. Nestas áreas ocorrem fundos coesivos (argila ou conteúdo de argila a partir de 10\% em depósitos siltosos ou arenosos); portanto, o tamanho do grão e seu peso são insignificantes comparados às forças eletroquímicas atuantes entre os minerais, de modo que as partículas soldam-se e formam uma massa coesa (Raudkivi, 1990).

Neste fundo, situado entre as isóbatas de 1,6 a 2,60 m e em áreas mais abrigadas da ação de ondas, ocorre predominância de material fino, variando de silte médio a fino, sendo que os grãos argila não representam percentuais maiores que $18 \%$ devido à alta ação hidrodinâmica, uma vez que estas amostras estão classificadas nos grupos C IV e D IV, propostos por Pejrup (1988).

As características das amostras deste fundo denotam um grau de seleção que varia de pobre a muito pobremente selecionada e assimetria positiva a muito positiva. 0 percentual de matéria orgânica oscila entre 12 e 18\%, com um aumento gradual da concentração em áreas mais profundas, de composição lamosa, e próximas aos rios Três Forquilhas e Cardoso, onde ocorre um maior aporte de sedimentos devido às descargas fluviais (Fig. 5).

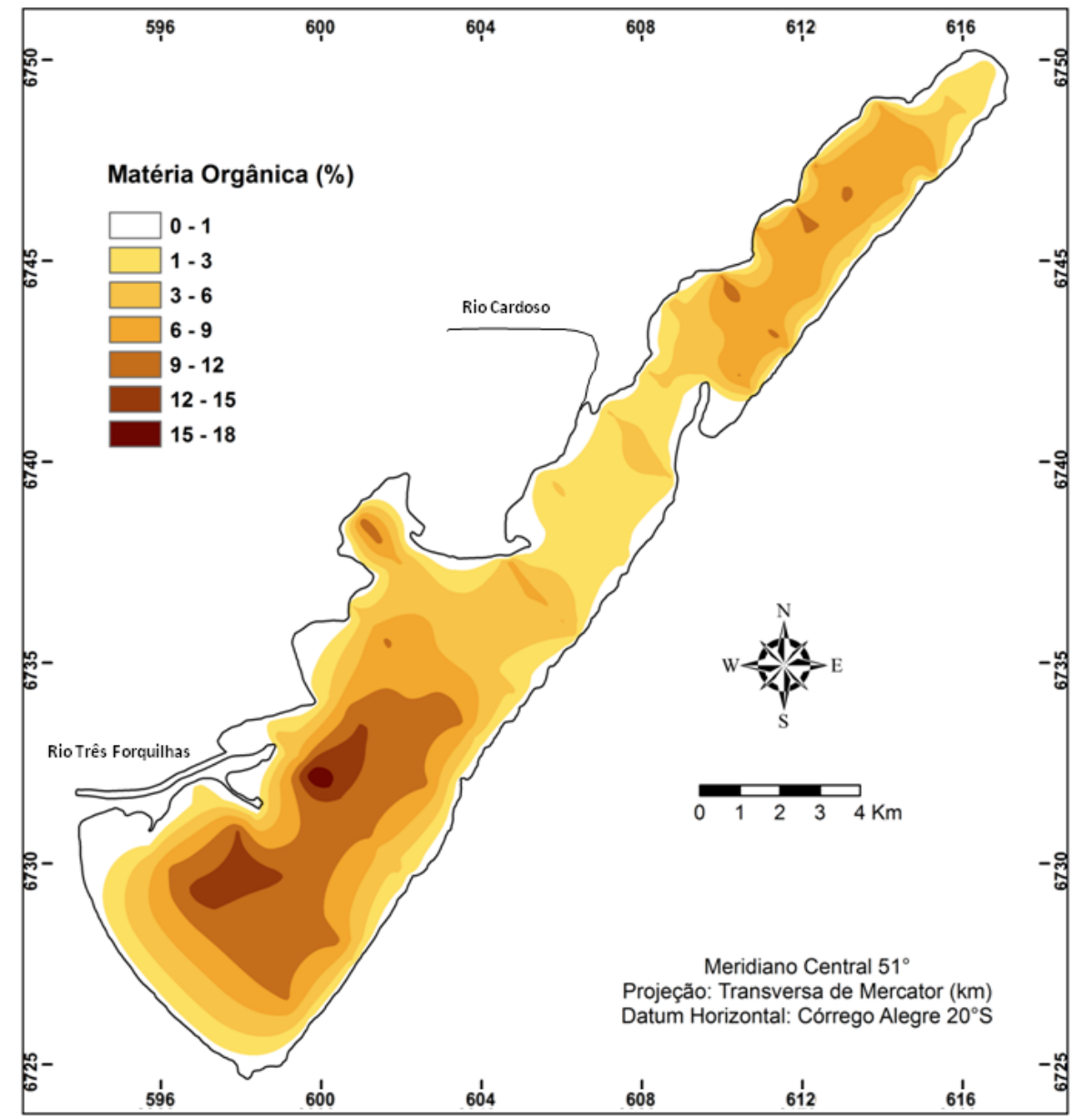

Figura 5. Mapa com os percentuais de matéria orgânica da Lagoa Itapeva. 


\subsection{Aporte sedimentar}

Os rios e córregos que escoam pela encosta do planalto (Fig. 1) são a fonte dominante de entrada de material sedimentar nas lagoas do litoral norte do Estado, transportando carga detrítica a partir das bacias de drenagem.

A perenidade da precipitação pluviométrica na região $(1300 \mathrm{~mm})$ é responsável por um transporte considerável de materiais clásticos para a Lagoa Itapeva, através da descarga dos rios Três Forquilhas e Cardoso, que apresentam vazões médias de $7,49 \mathrm{~m}^{3} \cdot \mathrm{s}^{-1}$ e $0.39 \mathrm{~m}^{3} . \mathrm{s}^{-1}$, com estimativa do aporte de sedimentos suspensos à lagoa de 47.380 Ton.ano $^{-1}$ e 5.740 Ton.ano $^{-1}$, respectivamente (ECOPLAN, 1997).

As rochas fonte de sedimentos que ingressam para o interior lacustre provêm da Formação Serra Geral, composta por rochas vulcânicas basálticas que extravasaram a partir do Período Jurássico e, secundariamente, rochas do arenito Botucatu, ambas pertencentes à Bacia do Paraná. Os rios que escoam pelo planalto transferem carga sedimentar na forma de pluma de sedimentos em suspensão (silte e argila), além de carga de tração composta de material de granulometria grossa (areias). Tanto a fauna como a flora lacustre bentônica contribuem com material biodetrítico de granulometria grossa para a lagoa.

Devido à perda da competência e capacidade destes tributários como agente transportador, ao ingressarem na lagoa, os sedimentos mais grossos são ali retidos, como exemplifica a foz do rio Três Forquilhas, que ostenta um sistema deposicional deltaico bem desenvolvido que prograda para o interior do ecossistema lacustre (Fig. 1). 0 delta apresenta uma extensão de aproximadamente $5 \mathrm{~km}$ e avança ativamente, de- senvolvendo uma planície deltaica subárea (Tomazelli \& Villwock, 1991).

A granulometria dos sólidos suspensos inseridos pelos rios Três Forquilhas e Cardoso na Lagoa Itapeva foi estimada e os resultados indicaram predomínio do tamanho silte e, com menor participação, argila e areia (Tab. 1). Sendo assim, os valores de argila inserida pelos rios por apresentarem um percentual menor em relação ao tamanho silte contribui para a pouca concentração desta classe granulométrica nas amostras de fundo.

0 interior lacustre também apresentou granulometria com baixo percentual de argila e areia nas porções norte e sul em grande parte devido à influência da carga transportada pelos rios (Tab. 1). Estes resultados encontrados na Lagoa Itapeva assemelham-se à do material em suspensão das águas superficiais da Lagoa dos Patos, que indicam como sendo $85 \%$ do material em suspensão constituído de silte, $10 \%$ de argila e $5 \%$ ou menos de areia (Hartmann \& Schettini, 1991).

Com relação à concentração de sólidos suspensos (CSS) contidos na água da Lagoa Itapeva, o interior la-

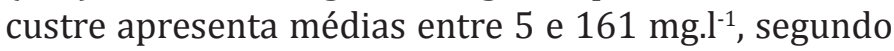
estudo realizado por Cardoso \& Motta Marques (2009). Comparativamente os valores de CSS na Lagoa Itapeva são próximos ao encontrado no estuário da Chesapeake Bay - USA, de 10 a 150 mg.l $\mathrm{l}^{-1}$, mas considerado elevado em relação aos valores no estuário da Gironde - França, de até 3 mg. ${ }^{-1}$ (Nichols \& Biggs 1985). Por outro lado, a concentração média é semelhante a outros corpos aquosos do estado, como no sistema lagunar Patos, que apresenta uma CSS entre 50 e $150 \mathrm{mg.l}^{-1}$ (Toldo Jr. et al., 2006) e ao Guaíba, com média de 35 mg. ${ }^{-1}$, e concentração máxima de $150 \mathrm{mg} \cdot \mathrm{l}^{-1}$ (Andrade Neto et al., 2012).

Tabela 1. Granulometria dos sólidos suspensos na Lagoa Itapeva e nos rios Três Forquilhas e Cardoso, onde (S) = Superfície, (M) = Meio e (F) = Fundo.

\begin{tabular}{llll}
\hline Local & \% Areia & \% Silte & \% Argila \\
\hline Rio Três Forquilhas (S) & 2,45 & 69,82 & 27,72 \\
Rio Três Forquilhas (M) & 2,94 & 65,72 & 31,33 \\
Rio Três Forquilhas (F) & 3,40 & 60,29 & 36,31 \\
& & & \\
Rio Cardoso (S) & 0,00 & 71,89 & 28,11 \\
Rio Cardoso (M) & 0,00 & 74,29 & 25,71 \\
Rio Cardoso (F) & 0,39 & 89,76 & 9,84 \\
& & & \\
Lagoa Itapeva Norte (S) & 0,00 & 70,09 & 25,39 \\
Lagoa Itapeva Norte(M) & 4,51 & 78,36 & 17,91 \\
Lagoa Itapeva Norte (F) & 5,40 & 74,73 & 19,87 \\
& & & \\
Lagoa Itapeva Sul (S) & 2,77 & 82,51 & 14,71 \\
Lagoa Itapeva Sul (M) & 1,65 & 88,11 & 10,24 \\
Lagoa Itapeva Sul (F) & 1,03 & 85,56 & 13,40 \\
\hline
\end{tabular}




\subsection{Forçantes da circulação lacustre}

A Lagoa Itapeva pertence ao padrão de lagoas do tipo "estrangulada", o qual tem sua circulação influenciada principalmente por ventos e descarga fluvial sem grande influência da maré, experimentando limitada variabilidade de curto prazo (Kjerfve, 1986). 0 regime eólico neste cenário constitui o principal fator hidrodinâmico responsável pela indução de ondas e correntes influenciando na deposição sedimentar.

0 vento consequentemente desempenha papel fundamental no controle da morfologia interna destas lagoas costeiras, induzindo o crescimento de pontais arenosos, bem como promovendo alterações em relação ao nível de água. Se o vento persistir por longos períodos, associado à elevada intensidade, pode ocasionar ondas do tipo seiches ao longo do eixo da lagoa, alterando o nível d'água em cada extremidade em um
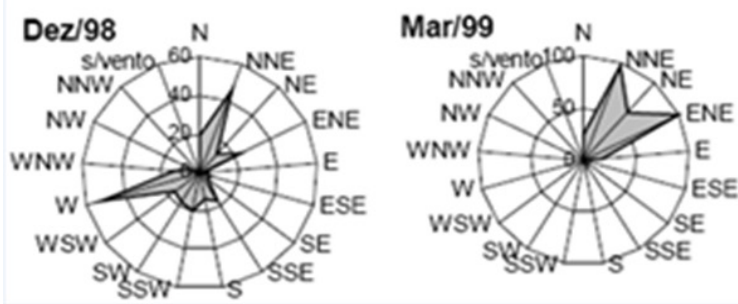

curto período de tempo.

O sistema eólico na área de estudo é dominado por um regime de alta energia e baixa variabilidade direcional (Medeiros, 1992). Dados referentes às direções de ventos na Lagoa Itapeva, em campanhas amostrais realizadas entre dezembro de 1998 a agosto de 1999 por Cardoso et al. (2003), revelaram um predomínio direcional que oscila do quadrante $\mathrm{N}$ e NE durante os meses de verão e ventos de W e SW durante os meses de inverno, acompanhando a passagem dos sistemas frontais (Fig. 6).

A ação hidrodinâmica induzida pelas ondas e sua relação com a distribuição dos sedimentos no piso lacustre foi estimada com a utilização do modelo teórico de predição de ondas do USACE (1984), sendo que os valores referentes à direção e frequência dos ventos foram obtidos de Cardoso et al. (2003), conforme tabela 2.
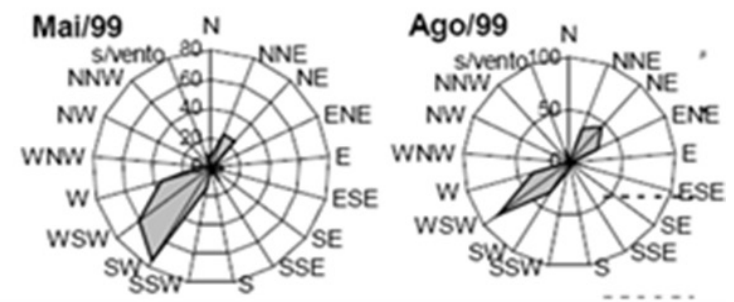

Figura 6. Frequência percentual (\%) da direção dos ventos na Lagoa Itapeva referente ao período de dezembro de 1998 a agosto de 1999 , modificado de Cardoso et al. (2003).

Tabela 2. Parâmetros relativos à formação de ondas e velocidade orbital produzidos pelo vento na Lagoa Itapeva.

\begin{tabular}{|c|c|c|c|c|c|c|c|c|}
\hline \multicolumn{4}{|c|}{ Vento } & \multirow{2}{*}{$\begin{array}{c}\text { Pista } \\
\mathrm{Km}\end{array}$} & \multicolumn{2}{|c|}{ Onda } & \multicolumn{2}{|c|}{ Velocidade Orbital (m.s $\left.{ }^{-1}\right)$} \\
\hline Estação & Direção & Frequência \% & Velocidade $(\mathrm{m} / \mathrm{s})$ & & Altura (m) & Período (s) & Superfície & Fundo $(2,10 \mathrm{~m})$ \\
\hline Sul & $\mathrm{N}$ & 16,8 & 2 & 9,6 & 0,1 & 1,2 & 0,28 & 0,00 \\
\hline Sul & $\mathrm{N}$ & 16,8 & 8 & 9,6 & 0,3 & 2,1 & 0,47 & 0,13 \\
\hline Sul & $\mathrm{N}$ & 16,8 & 16 & 9,6 & 0,5 & 2,7 & 0,61 & 0,30 \\
\hline Sul & NNE & 11 & 2 & 9,7 & 0,1 & 1,2 & 0,28 & 0,00 \\
\hline Sul & NNE & 11 & 8 & 9,7 & 0,3 & 2,1 & 0,47 & 0,13 \\
\hline Sul & NNE & 11 & 16 & 9,7 & 0,5 & 2,7 & 0,61 & 0,30 \\
\hline Sul & $\mathrm{NE}$ & 12,7 & 2 & 31 & 0,1 & 1,3 & 0,28 & 0,00 \\
\hline Sul & $\mathrm{NE}$ & 12,7 & 8 & 31 & 0,4 & 2,6 & 0,50 & 0.23 \\
\hline Sul & $\mathrm{NE}$ & 12,7 & 16 & 31 & 0,6 & 3,3 & 0,59 & 0,34 \\
\hline Sul & ENE & 9,2 & 2 & 12 & 0,1 & 1,2 & 0,29 & 0,00 \\
\hline Sul & ENE & 9,2 & 8 & 12 & 0,3 & 2,2 & 0,45 & 0,14 \\
\hline Sul & ENE & 9,2 & 16 & 12 & 0,5 & 2,8 & 0,59 & 0,31 \\
\hline Sul & $\mathrm{E}$ & 3,9 & 2 & 9,2 & 0,1 & 1,2 & 0,28 & 0,00 \\
\hline Sul & E & 3,9 & 8 & 9,2 & 0,3 & 2,1 & 0,47 & 0,13 \\
\hline Sul & E & 3,9 & 16 & 9,2 & 0,5 & 2,7 & 0,61 & 0,30 \\
\hline Norte & S & 6,6 & 2 & 9,6 & 0,1 & 1,2 & 0,28 & 0,00 \\
\hline Norte & S & 6,6 & 8 & 9,6 & 0,3 & 2,1 & 0,47 & 0,13 \\
\hline Norte & S & 6,6 & 16 & 9,6 & 0,5 & 2,7 & 0,61 & 0,30 \\
\hline Norte & SSW & 10,6 & 2 & 9,7 & 0,1 & 1,2 & 0,28 & 0,00 \\
\hline Norte & SSW & 10,6 & 8 & 9,7 & 0,3 & 2,1 & 0,47 & 0,13 \\
\hline Norte & SSW & 10,6 & 16 & 9,7 & 0,5 & 2,7 & 0,61 & 0,30 \\
\hline Norte & SW & 11,1 & 2 & 31 & 0,1 & 1,3 & 0,26 & 0,00 \\
\hline Norte & SW & 11,1 & 8 & 31 & 0,4 & 2,6 & 0,50 & 0,23 \\
\hline Norte & SW & 11,1 & 16 & 31 & 0,6 & 3,3 & 0,59 & 0,34 \\
\hline
\end{tabular}


Através da velocidade orbital das partículas, pode-se obter a relação entre a energia das ondas e o início da movimentação dos sedimentos de fundo. Tal procedimento é deduzido a partir de medidas de altura $(\mathrm{H})$ e período (T) da onda de superfície. Desta forma, a efetividade da onda em provocar o início do movimento dos sedimentos se dá em função da velocidade orbital próxima ao fundo e da sua frequência $(1 / \mathrm{T})$.

Baseado na simulação, a modelagem demonstrou que os ventos mais frequentes com média de intensidade de $16 \mathrm{~m} \cdot \mathrm{s}^{-1}$, atuando em uma pista de até $31 \mathrm{~km}$, geram ondas com altura significativa de até $0,60 \mathrm{~m}$ e período de 3,3 s. Esta intensidade de ventos provoca uma velocidade orbital de $0,34 \mathrm{~m} \cdot \mathrm{s}^{-1}$ junto ao piso lacustre (Tab. 2).

Trabalhos realizados por Hallermeier, (1980, apud USACE, 1984) demonstraram que a velocidade orbital junto ao fundo requerida para o início do movimento de sedimentos arenosos, com diâmetro entre 0,1 e 2,0 $\mathrm{mm}$, é de $0,13 \mathrm{~m} \cdot \mathrm{s}^{-1}$. Sendo assim, ventos com $16 \mathrm{~m} \cdot \mathrm{s}^{-1}$ ocasionam velocidades orbitais de $0,34 \mathrm{~m} \cdot \mathrm{s}^{-1}$ para uma profundidade de $2,10 \mathrm{~m}$. Como este valor excede o limite para movimentação de sedimentos tamanho areia, justifica-se o predomínio dessa classe granulométrica juntamente com o silte e a baixa concentração de grãos tamanho argila.

Portanto, grande parte dos sedimentos finos introduzidos pelos rios não encontram condições físicas para deposição no piso lacustre e seguem a descarga natural do Rio Cornélios em direção à Lagoa dos Quadros e à Laguna de Tramandaí, antes de serem transportados para o oceano (Fig. 7).

À medida que o fluxo de sedimentos em suspensão segue em direção à Laguna de Tramandaí, o efeito da intrusão salina oferece condições mais propícias à deposição dos sedimentos argilosos; isso ocorre em função do fenômeno de floculação, que ocasiona nos sedimentos finos a formação de flocos ou agregados, variando assim sua velocidade de sedimentação em algumas ordens de grandeza (Droppo \& Ongley, 1992). Segundo resultados apresentados por Tabajara (1994), a concentração de argila nas amostras de sedimentos de fundo atinge valores de até $66 \%$, demonstrando que o ambiente lagunar de Tramandaí oferece condições mais favoráveis à deposição de argilas provenientes das lagoas Itapeva e Quadros.

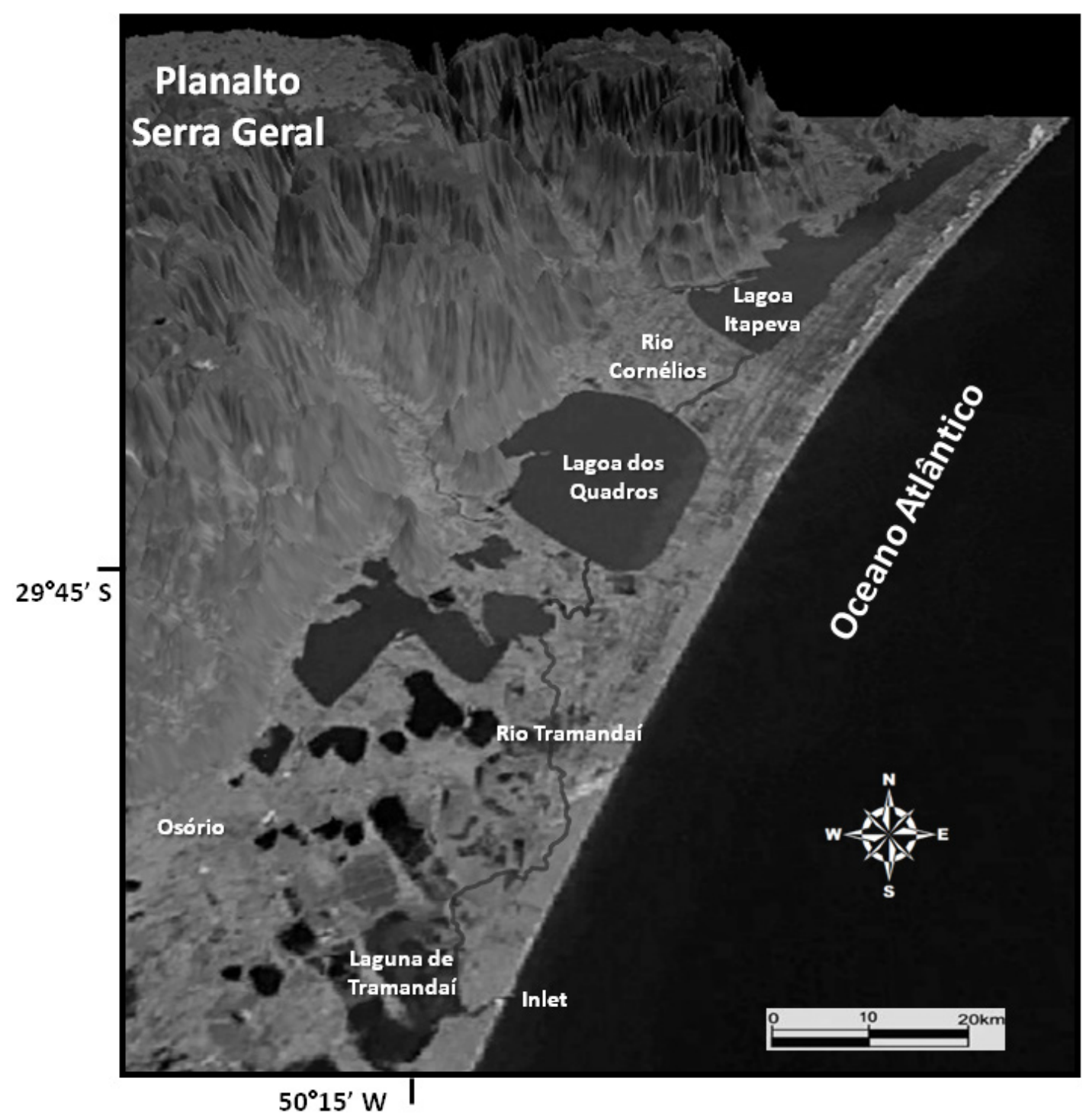

Figura 7. Fusão de imagem Landsat Thematic Mapper (TM) com MDT das lagoas interligadas pelos rios Cornélios e Tramandaí no Litoral Norte do RS, com destaque para a Lagoa Itapeva, Lagoa dos Quadros e Laguna de Tramandaí. 


\section{Conclusões}

Os sedimentos que recobrem o fundo da Lagoa Itapeva estão distribuídos segundo arranjos batimétricos, onde a profundidade reduzida determina a ocorrência de fundos mais expostos à turbulência das ondulações, na qual o elevado grau de energia dificulta a deposição das classes granulométricas finas, principalmente argila.

Nas áreas onde existe predomínio de sedimentos lamosos, ocorre um aumento gradativo do teor de matéria orgânica, principalmente em áreas próximas à desembocadura dos rios Três Forquilhas e Cardoso.

Os sedimentos das fácies sílticas estão associados às regiões abrigadas da ação dos ventos e às maiores profundidades, onde a menor velocidade orbital junto ao fundo favorece sua deposição.

0 vento exerce papel importante na circulação hidrodinâmica, formando ondas e correntes, que devido à baixa profundidade da lagoa, são capazes de agitar toda a extensão do fundo lacustre. A remobilização dos sedimentos do fundo mantém em suspensão grande parte da carga de granulometria fina, a qual em função da elevada energia hidrodinâmica, não encontra condições para deposição, seguindo pelos escoamentos naturais até a laguna de Tramandaí e depositando devido à floculação ou sendo exportada para o oceano.

A baixa concentração de argilas nas amostras de fundo ocorre tanto devido a menor inserção desta classe granulométrica pelos tributários, como também em decorrência da velocidade orbital junto ao fundo, que dificulta sua deposição.

O diagrama de Pejrup (1988) mostrou-se uma ferramenta importante na interpretação da distribuição sedimentar da Lagoa Itapeva, determinando a predominância em quase toda a extensão de uma hidrodinâmica elevada a muito elevada, justificando a reduzida concentração de grãos de diâmetro argila nas amostras de fundo.

Agradecimentos - Os autores agradecem a CAPES (Coordenação de Aperfeiçoamento de Pessoal de Nível Superior) pela bolsa de pesquisa e ao Centro de Estudos de Geologia Costeira e Oceânica - CECO da UFRGS, pelas facilidades e pelo apoio ao desenvolvimento deste trabalho.

\section{Referências}

Alves, A.R., Wassermam, J.C.F.A. \& Fernandez, G.B. 2006. Potencial de formações de esporões em lagunas. In: SIMPÓSIO NACIONAL DE GEOMORFOLOGIA, 6, Goiânia. Anais... Goiânia, v.1, p. 1-8.

Andrade Neto, J.S., Rigon, L.T., Toldo Jr., E.E. \& Schettini, C.A.F. 2012. Descarga sólida em suspensão do sistema fluvial do Guaíba, RS, e sua variabilidade temporal. Pesquisas em Geociências, 39(2): 161-171.

Angulo R.J. \& Lessa G.C. 1997. The Brazilian sea level curves: a critical review with emphasis on the curves from Paranaguá and Cananéia regions. Marine Geology, 140: 141166.
Camargo, M.G. 2006. SysGran: um sistema de código aberto para análises granulométricas do sedimento. Revista Brasileira de Geociências, 36(2): 371-378.

Cardoso, L., Silveira, A.L.L. da S. \& Motta Marques, D.M.L. 2003. A ação do vento como gestor da hidrodinâmica na Lagoa Itapeva (litoral norte do Rio Grande do Sul). Revista Brasileira de Recursos Hídricos, 8 (3): 5-15.

Cardoso, L.S. \& Motta Marques, D.M.L. 2009. Hydrodynamics-driven plankton community in a shallow lake. Aquatic Ecology, 43 (1): 73-84.

Corrêa, I.C.S. \& Toldo Jr., E.E. 1998. The Sea Level Stabilization In The Rio Grande do Sul Continental Shelf, Brazil. Anais da Academia Brasileira de Ciências, 70 (2): 213-219.

Dillenburg S.R., Roy, P.S., Cowell, P.J. \& Tomazelli, L.J. 2000. Influence of antecedent topography on coastal e v o l u tion as tested by shoreface translation barrier model (STM). Journal of Coastal Research, 16 (1): 71-81.

Dillenburg, S.R., Tomazelli, L.J., Hesp, P.A., Barboza, E.G., Clerot, L.C.P. \& Silva, D.B. 2006.Stratigraphy and evolution of a prograded transgressive dunefield barrier in southern Brazil. Journal of Coastal Research, 39: 132-135.

Droppo I.G. \& Ongley E.D., 1992, The state of suspended sediment in the freshwater fluvial environment: a method of analysis, Water Research, 26 (1): 65-72.

ECOPLAN Engenharia Ltda. 1997. Avaliação da disponibilidade hídrica superficial e subterrânea do litoral norte do Rio Grande do Sul, englobando todos os corpos hídricos que drenam para o Rio Tramandaí. Relatório Técnico Final. São Paulo. ECOPLAN, 432p.

Folk, R.L. \& Ward, W.C. 1957. Brazos river bar: A study in the significance of grain size parameters. Journal of Sedimentary Petrology, 27: 3-27.

Folk, R.L., 1968. Petrology of Sedimentary Rocks. Hemphill's, Austin, pp. 170.

Hartmann, C. \& Schettini, C.A.F. 1991. Aspectos Hidrológicos na Desembocadura da Laguna dos Patos, RS. Revista Brasileira de Geociências, 21 (4): 371-377.

Hasenack, H. \& Ferraro, L.W. 1989. Considerações sobre o clima da região de Tramandaí, RS. Pesquisas em Geociências, 22: 53-70.

Kjerfve, B. 1986. Comparative oceanography of coastal lagoons. In: Wolfe D.A (ed) Estuarine variability. Academic Press, New York, pp 63-81.

Kjerfve, B., Ribeiro, C.H.A., Dias, G.T.M., Filippo, A. M. \& Quaresma, V.S. 1997. Oceanographic characteristics of an impacted coastal bay: baía de Guanabara, Rio de Janeiro, Brazil. Continental Shelf Research, 17 (13): 1609-1643.

Krumbein, W.C. \& Pettijohn, F.J. 1938. Manual of Sedimentary Petrography. New York: Appleton-Century-Crofts, 549p.

Martin L., Suguio K. \& Flexor, J.M. 1979. Le Quaternaire marin du littoral brésilien entre Cananéia (SP) et Barra de Guaratiba (RJ). In: INTERNATIONAL SYMPOSIUM ON COASTAL EVOLUTION IN THE QUATERNARY, Proceedings... São Paulo, Brasil, pp 296-331.

McManus, J. 1988. Grain size determination and interpretation. In: M.E. Tucker (ed.) Techniques in Sedimentology, Oxford, Blackwells, p.63-85.

Meyer, K.E.B., Souza, P.A., Cwik, M.R., Menezes, T.R. \& Buchmann, F.S. 2006. Palinofácies e Processos Deposicionais em Sedimentos de Fundo da Lagoa dos Quadros, Planície Costeira do Rio Grande do Sul, Brasil. Revista Brasileira de Geociências, 36 (4): 569-576.

Medeiros, P. R. P. 1992. Estudo do Sistema Lagunar-Estuarino de Tramandaí-Imbé: Física e Química da Água. Porto Ale- 
gre, 112p. Dissertação de Mestrado, Programa de Pós-graduação em Ecologia, Instituto de Biociências, Universidade Federal do Rio Grande do Sul.

Nichols, M., 1989. Sediment accumulation rates and relative sea-level rise in lagoons. Marine Geology, 88: 201-219.

Nichols, M.M. \& Biggs, R.B. 1985. Estuaries. In: Davies Jr., R.A.(ed.). Coastal Sedimentary Environments, SpringerVerlag, $716 \mathrm{p}$.

Pejrup, M. 1988. The triangular diagram used for classification of estuarine sediments a new approach. In: Boer, P.L.; van Gelder, A. \& Nio, S.D.(eds.). Tide-Influenced Sedimentary Environments and Facies. D. Reidel, Dordrencht. p.289-300.

Raudkivi, A.J. 1990. Loose Boundary Hydraulics.3. Ed. Pergamon Press. 539p.

Schäfer, A. 1988. Tipificação ecológica das lagoas costeiras do Rio Grande do Sul, Brasil. Acta Limnologica Brasiliensia, 2: 29-55.

Schwarzbold, A. \& Schäfer, A. 1984. Gênese e morfologia das Lagoas Costeiras do Rio Grande do Sul. Amazoniana, 9 (1): 87-104.

Strahler, A. N. \& Strahler, A. H. 1987. Modern Physical Geography. John Willey and Sons, New York. 488 p.

Tabajara, L.L.C.A. 1994. Aspectos Hidrodinâmicos e Sedimentologia do Sistema Lagunar-Estuarino de Tramandaí. Porto Alegre, 202p. Dissertação de Mestrado, Curso de Pós-Graduação em Geociências, Instituto de Geociências, Universidade Federal do Rio Grande do Sul.

Toldo Jr., E.E. 1998. Morfodinâmica da Laguna dos Patos, Rio
Grande do Sul. Pesquisas em Geociências 18(1): 58-63.

Toldo Jr., E.E. \& Medeiros R.K. 1986. Programa interpolar em linguagem basic para análise estatística e propriedades texturais de amostras sedimentares em computador. Pesquisas em Geociências, 18(1): 91-100.

Toldo Jr., E.E., Dillenburg, S.R., Corrêa, I.C.S. \& Almeida, L.E.S.B., 2000. Holocene Sedimentation in Lagoa dos Patos Lagoon, Rio Grande do Sul, Brazil. Jounal of Coastal Research, 16 (3): 816-822.

Toldo Jr., E. E., Dillenburg, S.R., Corrêa, I.C.S., Almeida, L.E.S.B., Weschenfelder, J. \& Gruber, N.L.S. 2006. Sedimentação de Longo e Curto Período na Lagoa dos Patos, Sul do Brasil. Pesquisas em Geociências, 33(2): 79-86.

Tomazelli, L. J. \& Villwock, J. A. 1991. Geologia do sistema lagunar holocênico do Litoral Norte do Rio Grande do Sul, Brasil. Pesquisas em Geociências, 18 (1): 13-24.

USACE (U.S Army Corps of Engineers). 1984. Shore Protection Manual. 4 ed. U.S. Government Printing Office, Washington, D.C.

Villwock, J.A. 1984. Geology of the coastal province of Rio Grande do Sul, Southern Brazil, a synthesis. Pesquisas em Geociências, 16: 5-49.

Villwock, J.A. \& Tomazelli, L.J. 1998. Holocene coastal evolution in Rio Grande do Sul. Brazil. Quaternary of South America and Antarctic Peninsula, 11: 283-296.

Wetzel, R.G. 1975. Limnology. W.B. Sauders Company, Philadelphia. 743p.

Zenkovitch V.P. 1959. On the genesis of cuspate spits along lagoon shores. Journal of Geology, 67 (3): 269-277.

Manuscrito 513.

Editores: Iran S. Corrêa \& César L. Schultz. 
